\title{
Reticular dysgenesis caused by an intronic pathogenic variant in AK2
}

\author{
Shoji Ichikawa, ${ }^{1}$ Susan Prockop, ${ }^{2,3}$ Charlotte Cunningham-Rundles, ${ }^{4}$ \\ Travis Sifers, ${ }^{4,8}$ Blair R. Conner, ${ }^{5}$ Sitao Wu, ${ }^{6}$ Rachid Karam, ${ }^{5}$ Michael F. Walsh, ${ }^{2,7}$ \\ and Elise Fiala ${ }^{2,7}$ \\ ${ }^{1}$ Department of Clinical Diagnostics, Ambry Genetics, Aliso Viejo, California 92656, USA; ${ }^{2}$ MSK Kids, \\ Department of Pediatrics, ${ }^{3}$ MSK Kids, Stem Cell Transplant and Cellular Therapy, Memorial Sloan Kettering \\ Cancer Center, New York, New York 10065, USA; ${ }^{4}$ Division of Clinical Immunology, Departments of Medicine \\ and Pediatrics, Icahn School of Medicine at Mount Sinai, New York, New York 10029, USA; ${ }^{5}$ Department of \\ Research and Development, ${ }^{6}$ Department of Bioinformatics and Computational Biology, Ambry Genetics, \\ Aliso Viejo, California 92656, USA; ${ }^{7}$ Clinical Genetics Service, Department of Medicine, Memorial Sloan \\ Kettering Cancer Center, New York, New York 10021, USA
}

Abstract Reticular dysgenesis is a form of severe combined immunodeficiency (SCID) caused by biallelic pathogenic variants in AK2. Here we present the case of a boy diagnosed with SCID following a positive newborn screen (NBS). Genetic testing revealed a homozygous variant: AK2 c. $330+5 G>A$. In silico analyses predicted weakened native donor splice site. However, this variant was initially classified as a variant of uncertain significance (VUS) given lack of direct evidence. To determine the impact on splicing, we analyzed RNA from the proband and his parents, using massively parallel RNA-seq of cloned RT-PCR products. Analysis showed that c. $330+5 G>$ A results in exon 3 skipping, which encodes a critical region of the AK2 protein. With these results, the variant was upgraded to pathogenic, and the patient was given a diagnosis of reticular dysgenesis. Interpretation of VUS at noncanonical splice site nucleotides presents a challenge. RNA sequencing provides an ideal platform to perform qualitative and quantitative assessment of intronic VUS, which can lead to reclassification if a significant impact on mRNA is observed. Genetic disorders of hematopoiesis and immunity represent fruitful areas to apply RNA-based analysis for variant interpretation given the high expression of RNA in blood.

Corresponding author: fialae@mskcc.org

(C) 2020 Ichikawa et al. This article is distributed under the terms of the Creative Commons Attribution-NonCommercial License, which permits reuse and redistribution, except for commercial purposes, provided that the original author and source are credited.

Ontology term: severe combined immunodeficiency

Published by Cold Spring Harbor Laboratory Press

doi:10.1101/mcs.a005017

\section{INTRODUCTION}

Reticular dysgenesis (MIM 267500) is one of the rarest and most severe forms of SCID. It is characterized by the combination of SCID with congenital agranulocytosis and bilateral sensorineural deafness. Impairment in lymphoid and myeloid lineages results in profound neutropenia (unresponsive to granulocyte colony stimulating factor; GCSF) along with T and natural killer (NK) cell lymphopenia in affected patients (Lagresle-Peyrou et al. 2009). Like other forms of SCID, treatment is with hematopoietic stem cell transplant.

Reticular dysgenesis is an autosomal recessive disorder caused by biallelic loss-of-function pathogenic variants in AK2 (MIM 103020) (Lagresle-Peyrou et al. 2009; Pannicke et al. 2009). AK2 encodes adenylate kinase 2 , an enzyme in the mitochondrial intermembrane space that catalyzes the reversible transfer of the terminal phosphate group between ATP

\footnotetext{
${ }^{8}$ Present affiliation: Center for Allergy and Immunology, Kansas City, Missouri 64111, USA
} 
and AMP. AK2 deficiency significantly impairs energy metabolism, leading to reduced hematopoietic stem, and progenitor cells (Hoenig et al. 2018).

Two intronic AK2 mutations, affecting canonical splice sites, have previously been reported in patients with reticular dysgenesis (Pannicke et al. 2009). Non-canonical splice site variants may also be predicted to affect splicing by in silico tools, although these variants often lack direct evidence and remain variants of unknown significance (VUS). RNA analysis has the potential to resolve these as abnormal RNA transcripts can be considered a very strong line of evidence supporting pathogenicity (Richards et al. 2015). Here we report the first noncanonical splice site pathogenic variant in $A K 2$, resulting in reticular dysgenesis in the homozygous state.

\section{RESULTS}

\section{Clinical Presentation}

This male patient of Sudanese ancestry with consanguineous parents presented to our Pediatric Clinical Genetics Service at $28 \mathrm{~d}$ of life (DOL) due to a positive NBS for SCID (<200 TRECS/ $\mu$ l blood) and follow-up testing consistent with SCID.

He was born full-term following an uncomplicated pregnancy and admitted to the NICU for respiratory distress. He was found to have severe neutropenia on DOL 1, which persisted despite receiving GCSF. On DOL 7, he had an episode of severe metabolic acidosis, and complete metabolic work-up was unremarkable.

Further evaluation demonstrated CD3 T cells of 37 cells/mcL (reference range 7692222), CD4 T cells of 31 cells/mcL (429-1131), CD4+45RA+ cells of 0 cells/mcL (89-789), CD8 T cells of 6 cells/mcL (209-768), CD19 B cells of 95 cells/mcL (85-441), NK cells of 62 cells/mcL (78-510), NK function of $4 \%$ cytotoxicity (17-78), phytohemagglutinin (PHA) of $59 \%$ of CD3 (77-100), IgA $<5 \mathrm{mg} / \mathrm{dL}$ (3-30), and $\operatorname{lgM}<5 \mathrm{mg} / \mathrm{dL}$ (10-90). There was no evidence of maternal engraftment revealed by testing of short tandem repeats within the T cell subset.

Other clinical features included small ventriculoseptal defects, an anorectal malformation with excessive perianal tissue, and short stature (tracking at or below third percentile for height). He passed his hearing newborn screening (NBS); however, a follow-up hearing evaluation was performed because of clinical concerns, and an auditory brainstem response revealed profound bilateral sensorineural hearing loss.

Initial genetic testing at GeneDx (Gaithersburg, MD) included a karyotype, which was normal, and a chromosome microarray, which detected regions of homozygosity consistent with reported consanguinity but no deletions or duplications. An 85-gene Comprehensive SCID and CID Panel (Invitae) revealed two homozygous and three heterozygous VUS, all in genes associated with autosomal recessive forms of immunodeficiency. The homozygous variants were AK2 c. $330+5 \mathrm{G}>\mathrm{A}$ and ATM (MIM 607585) c.5497-4C>G. Alpha-fetoprotein (AFP) levels were normal for age and downtrending, so the ATM variant was considered not clinically significant. The AK2 variant in intron 3 was suspicious, although at this time remained a VUS. The parents were both heterozygous for the AK2 variant. Given the patient's additional clinical features and known consanguinity, exome sequencing was subsequently sent to GeneDx and revealed an additional diagnosis of Stickler syndrome type V (MIM 614284) because of a homozygous COL9A2 (MIM 120260) likely pathogenic variant (c.1242delC; p.G415Efs*116). Details of the three variants are provided in Table 1.

At 3 mo old, the patient underwent peripheral blood stem cell transplant from a matched unrelated donor following cytoreduction with busulfan, fludarabine, and rabbit antithymocyte globulin (rATG) and engrafted. 


\begin{tabular}{|c|c|c|c|c|c|c|c|c|}
\hline Gene & Chromosome & $\begin{array}{l}\text { HGVS DNA } \\
\text { reference }\end{array}$ & $\begin{array}{l}\text { HGVS protein } \\
\text { reference }\end{array}$ & $\begin{array}{l}\text { Variant } \\
\text { type }\end{array}$ & $\begin{array}{l}\text { Predicted } \\
\text { effect }\end{array}$ & $\mathrm{dbSNP}$ & Genotype & Comments \\
\hline$A K 2$ & 1 & $\begin{array}{l}\text { NM_001625.3: } \\
\text { c.330 + 5G >A }\end{array}$ & & SNV & $\begin{array}{r}\text { Splicing } \\
\text { defect }\end{array}$ & & Homozygous & \\
\hline ATM & 11 & $\begin{array}{l}\text { NM_000051.3: } \\
\text { c.5497-4C > G }\end{array}$ & & SNV & Unknown & rs1002998981 & Homozygous & Normal AFP \\
\hline COL9A2 & 1 & $\begin{array}{l}\text { NM_001852.3: } \\
\text { c.1242delC }\end{array}$ & $\begin{array}{l}\text { NP_001843.1: } \\
\text { p.G415Efs*116 }\end{array}$ & Deletion & Frameshift & rs756694568 & Homozygous & \\
\hline
\end{tabular}

(SNV) Single-nucleotide variant, (AFP) alpha-fetoprotein.

\section{RNA-seq Analysis}

In silico analyses had predicted that $A K 2$ c. $330+5 G>A$ likely weakens the native donor site (Table 2). To directly determine the impact on splicing, we analyzed RNA from the proband and his unaffected parents. Electrophoresis of reverse transcriptase-polymerase chain reaction (RT-PCR) products showed that the proband lacks the normal transcript, whereas the parents have similar amounts of normal and truncated transcripts (Fig. 1A). Quantitation of RNA-seq results revealed that this variant results in near complete skipping of exon 3 (r.220_330del) in the proband and $~ 50 \%$ skipping in his parents (Fig. 1B,C). The loss of exon 3 is predicted to result in an in-frame deletion of 37 amino acids, which account for $15 \%$ (37/239 amino acids) of the AK2 protein. The proband also had small amounts of transcripts missing exons 2 and 3 (r.94_330del), which were absent in other samples (data not shown).

\section{Variant Reclassification}

Variant classification was then revisited. Final classification was made based on our internal variant assessment scheme, as well as ACMG guidelines, which apply evidence based on the type and strength of the criteria for pathogenicity—Pathogenic Supporting (PP), Pathogenic Moderate (PM), Pathogenic Strong (PS), and Pathogenic Very Strong (PVS) (Richards et al. 2015). This variant is absent in gnomAD (PM2), and all in silico tools predict weakening of the native donor site (PP3). The phenotype was consistent with reticular dysgenesis (PP4_Moderate).

RNA results could be considered strong evidence (PS3) per ACMG guidelines. However, because they provided exact molecular consequence of this variant (exon 3 skipping), we used recommended PVS1 criterion for deletion of an exon (Abou Tayoun et al. 2018). The deleted region (p.V74_M110del) located in the adenylate kinase domain (Lagresle-Peyrou et al. 2009) is likely critical for catalytic activity. The presence of exon 3 in almost all known

Table 2. In silico analyses of $A K 2$ c. $330+5 G>A$

\begin{tabular}{lccc}
\hline Prediction tool & Reference (G) score & Variant (A) score & Variation (\%) \\
\hline NNSplice (Reese et al. 1997) & 0.43 & 0.03 & -93.02 \\
ESEfinder (Cartegni et al. 2003) & 9.17 & 5.73 & -37.51 \\
Human Splicing Finder (Desmet et al. 2009) & 84.80 & 72.63 & -14.35 \\
MaxEnt (Yeo and Burge 2004) & 6.18 & -1.33 & -121.52 \\
SpliceAl (Jaganathan et al. 2019) & N/A & DL 0.91 & N/A \\
\hline
\end{tabular}

aprobability of donor site loss. 

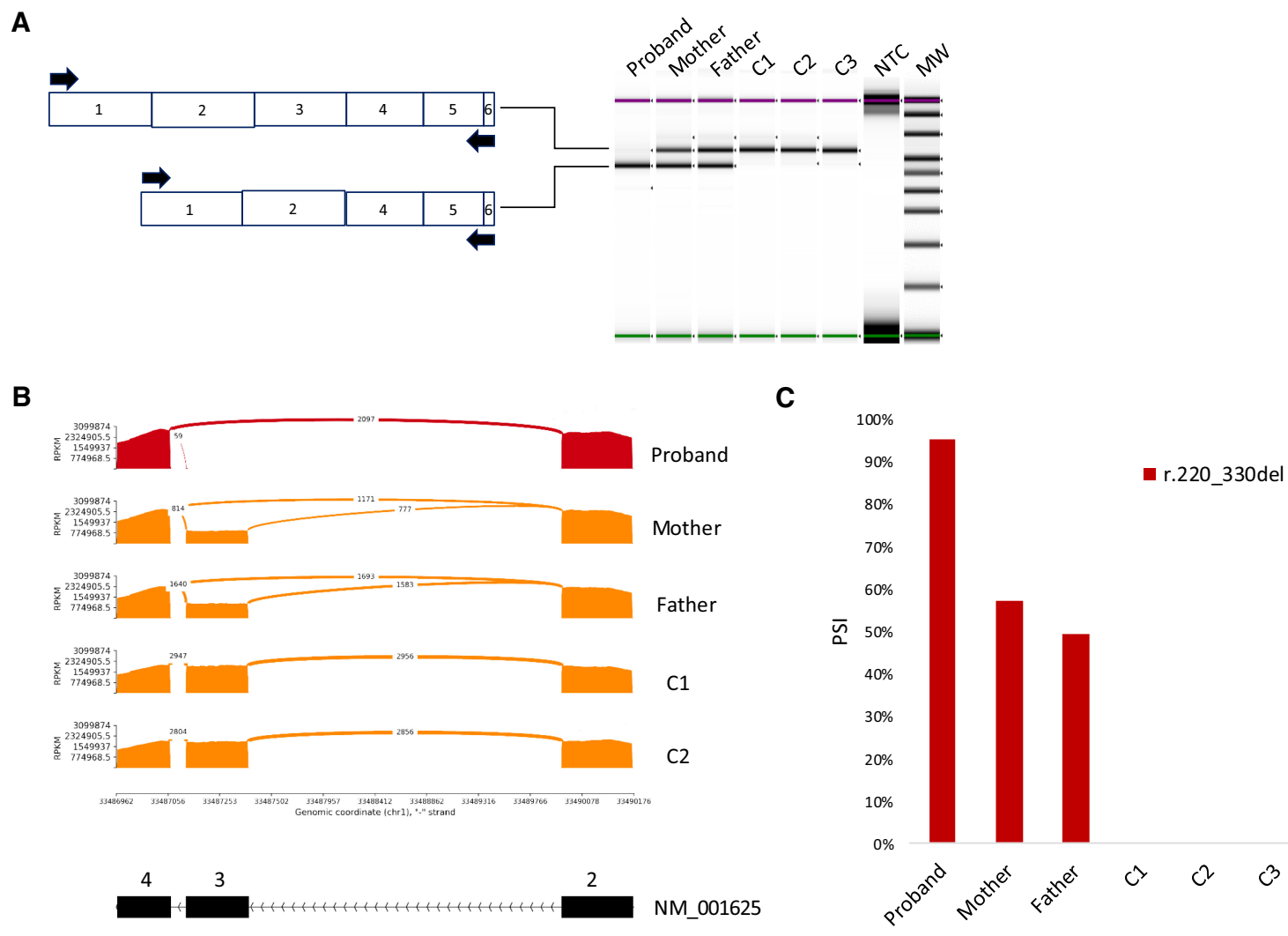

Figure 1. Aberrant splicing caused by $A K 2$ c. $330+5 G>A$. (A) TapeStation electrophoresis of reverse transcriptase-polymerase chain reaction (RT-PCR) products with the schematic of predicted mRNA. Locations of the primers are shown by arrows. (C1 and C2) Negative controls (blood), (C3) negative control (colon), (NTC) no template control, (MW) molecular weight. (B) Sashimi plot of AK2 exons 2-4 from CloneSeq, showing that the majority of reads in the proband do not harbor exon 3. RefSeq AK2 isoform (NM_001625) is shown at the bottom. (C) Relative quantification of abnormal transcripts shown in B: r.220_330del (skipping of exon 3). (PSI) Percent spliced in index.

alternate isoforms suggests that the region encoded by this exon is functionally important. One affected patient has been reported with a pathogenic missense variant in this region (p.Arg103Trp) that resulted in markedly lower levels of AK2 protein (Lagresle-Peyrou et al. 2009). Although the deletion likely impacts critical function of the protein, residual protein function cannot be ruled out because the deletion is in-frame. Thus, PVS1_Strong was assigned to the RNA evidence. With this evidence, the variant was reclassified to pathogenic based on our classification scheme or likely pathogenic by ACMG—one strong, two moderate, and one supporting.

\section{DISCUSSION}

Noncanonical splice site variants present a challenge for interpretation and often remain as unresolved VUS. In silico tools have limited ability to accurately predict effects on splicing. As presented here, RNA-seq provides an ideal platform for qualitative and quantitative assessment of intronic VUS, which can lead to reclassification if a significant impact on mRNA (or lack thereof) is observed. The RNA analysis we performed on AK2 c.330+5G > A revealed 
that this variant results in almost complete absence of normal transcripts, leading to its reclassification as (likely) pathogenic. Most importantly, this reclassification allowed us to provide a definitive diagnosis to the family.

One limitation of performing RNA-based analysis in many cases is the procurement of tissues that express the gene of interest. Many genes have little to no expression in blood or other readily available tissues. Being involved in early hematopoietic stem cell survival and function (Hoenig et al. 2018), AK2 is highly expressed in hematopoietic tissue, allowing us to collect samples needed for the study. This and other genetic disorders of hematopoiesis and immunity may represent one of the more fruitful areas to apply RNA-based analysis for variant interpretation given the high gene expression in blood.

In addition to a unique hematological phenotype, reticular dysgenesis is associated with sensorineural hearing loss. Although Stickler syndrome likely also contributes to our patient's hearing loss, his profound hearing loss across all frequencies tested is beyond what would be expected because of Stickler syndrome alone, which results in high-frequency loss (Nixon et al. 2019). Lactic acidosis has not been previously described as a manifestation of reticular dysgenesis; however, given AK2's role in metabolism and lactate production (Six et al. 2015), it is possible that our patient's lactic acidosis was secondary to AK2 deficiency as opposed to an occult infection.

Amended results were provided to the family following transplant. In addition to providing a definitive diagnosis, the pathogenic result can be used for testing of siblings in the future. Reticular dysgenesis has specific implications for conditioning prior to stem cell transplant (Hoenig et al. 2018), and therefore making this diagnosis very early in life will be important for management of any future siblings or other relatives who may share the homozygous $A K 2$ variant.

In summary, we identified the first pathogenic noncanonical splicing variant in AK2 in a patient with reticular dysgenesis. This highlights the utility and importance of RNA-based analysis for splicing.

\section{METHODS}

Massively Parallel RNA-seq Analysis

Peripheral blood samples were collected from the proband (prior to transplant) and his parents in PAXgene Blood RNA Tubes (QIAGEN). RNA isolation was performed on the QIAcube (QIAGEN) using the PAXgene Blood RNA Kit (PreAnalytiX). Massively parallel RNA-seq of cloned RT-PCR products (CloneSeq) was performed as described previously (Farber-Katz et al. 2018). Briefly, $300 \mathrm{ng}$ of total RNA input was used to generate cDNA using the SuperScript IV kit and Oligo(dT) (QIAGEN). Primers spanning introns were custom designed: 5'-CGAACTGGTGGCAGTGAGA-3' in exon 1 and 5'-GTTCCCCGGTGATGTCAT-3' in exons $5 / 6$ (Figure 1A). Three independent RT-PCRs performed in proband, parents, and healthy blood and colon controls were pooled together, and size was determined using TapeStation D1000 reagents (Agilent). RT-PCR products were cloned using the pGEM-T Easy kit (Promega), and isolated plasmids underwent library prep using standard illumina dual index adapters. Paired-end sequencing was performed on MiSeq (illumina) using 250-bp reads.

\section{Bioinformatics Analysis}

Paired-end reads were aligned to the human genome (GrCh37/hg19) using STAR aligner v2.5.2a. Aligned reads were analyzed using a custom bioinformatics pipeline capable of detecting exon skipping, partial exon skipping, partial intron inclusion, intron retention, and the 
Competing Interest Statement

S.I., B.R.C., S.W., and R.K. are employees of Ambry Genetics.

\section{Referees}

Xiaowu Gai

Andrey V. Marakhonov

Anonymous

Received November 25, 2019; accepted in revised form March 23, 2020. presence of a cryptic exon as described previously (Farber-Katz et al. 2018). The percentage of alternative splicing events was calculated relative to the primary AK2 isoform (NM_001625) by dividing the number of reads supporting the splicing event by the total number of reads in the region of the splicing event. These values were normalized according to read length and exon length to determine percent spliced in (PSI) as described previously (Schafer et al. 2015). Sashimi plots depicting the total reads spanning exons 2 to 5 were generated using Mixture of Isoforms software (MISO). Sashimi plots provide a quantitative visualization of aligned CloneSeq reads, enabling quantitative comparison of exon usage across samples (Katz et al. 2015).

\section{Variant Classification}

Pathogenicity of the AK2 variant was reassessed to incorporate RNA results. Classification is according to the classification scheme established by our laboratory (Pesaran et al. 2016), which incorporates ACMG guidelines (Richards et al. 2015) and was updated with recommended use of PVS1 criterion for loss-of-function variants (Abou Tayoun et al. 2018). As a comparison, the variant was also reassessed using the ACMG guidelines.

\section{ADDITIONAL INFORMATION}

\section{Data Deposition and Access}

The AK2 variant and our interpretation have been submitted to ClinVar (https://www.ncbi .nlm.nih.gov/clinvar/) and can be found under accession number SCV001244679. RNA sequence data that support the findings of this study are available on request from the corresponding author (E.F.). The patient data are not publicly available because they contain information that could compromise research participant privacy/consent.

\section{Ethics Statement}

The patient's parents gave informed consent for all clinical testing and research participation. This study was approved by the Western Institutional Review Board.

\section{Acknowledgments}

We thank our patient's family for their participation.

\section{Author Contributions}

E.F., M.F.W., S.P., C.C.-R. and T.S. were involved in the clinical care of this patient. S.I., B.R.C., S.W., and R.K. performed the RNA analysis and variant interpretation. S.I. and E.F. prepared the manuscript, which all authors participated in editing.

\section{Funding}

M.F.W. is supported by the Robert and Kate Niehaus Center for Inherited Cancer Genomics at Memorial Sloan Kettering.

\section{REFERENCES}

Abou Tayoun AN, Pesaran T, DiStefano MT, Oza A, Rehm HL, Biesecker LG, Harrison SM; ClinGen Sequence Variant Interpretation Working Group. 2018. Recommendations for interpreting the loss of function PVS1 ACMG/AMP variant criterion. Hum Mutat 39: 1517-1524. doi:10.1002/humu.23626 
Cartegni L, Wang J, Zhu Z, Zhang MQ, Krainer AR. 2003. ESEfinder: a web resource to identify exonic splicing enhancers. Nucleic Acids Res 31: 3568-3571. doi:10.1093/nar/gkg616

Desmet FO, Hamroun D, Lalande M, Collod-Beroud G, Claustres M, Beroud C. 2009. Human splicing finder: an online bioinformatics tool to predict splicing signals. Nucleic Acids Res 37: e67. doi:10.1093/nar/ gkp215

Farber-Katz S, Hsuan V, Wu S, Landrith T, Vuong H, Xu D, Li B, Hoo J, Lam S, Nashed S, et al. 2018. Quantitative analysis of $B R C A 1$ and $B R C A 2$ germline splicing variants using a novel RNA-massively parallel sequencing assay. Front Oncol 8: 286. doi:10.3389/fonc.2018.00286

Hoenig M, Pannicke U, Gaspar HB, Schwarz K. 2018. Recent advances in understanding the pathogenesis and management of reticular dysgenesis. Br J Haematol 180: 644-653. doi:10.1111/bjh.15045

Jaganathan K, Kyriazopoulou Panagiotopoulou S, McRae JF, Darbandi SF, Knowles D, Li YI, Kosmicki JA, Arbelaez J, Cui W, Schwartz GB, et al. 2019. Predicting splicing from primary sequence with deep learning. Cell 176: 535-548. doi:10.1016/j.cell.2018.12.015

Katz Y, Wang ET, Silterra J, Schwartz S, Wong B, Thorvaldsdóttir H, Robinson JT, Mesirov JP, Airoldi EM, Burge CB. 2015. Quantitative visualization of alternative exon expression from RNA-seq data. Bioinformatics 31: 2400-2402. doi:10.1093/bioinformatics/btv034

Lagresle-Peyrou C, Six EM, Picard C, Rieux-Laucat F, Michel V, Ditadi A, Demerens-de Chappedelaine C, Morillon E, Valensi F, Simon-Stoos KL, et al. 2009. Human adenylate kinase 2 deficiency causes a profound hematopoietic defect associated with sensorineural deafness. Nat Genet 41: 106-111. doi:10.1038/ ng.278

Nixon TRW, Alexander P, Richards A, McNinch A, Bearcroft PWP, Cobben J, Snead MP. 2019. Homozygous type IX collagen variants (COL9A1, COL9A2, and COL9A3) causing recessive Stickler syndrome-expanding the phenotype. Am J Med Genet 179: 1498-1506. doi:10.1002/ajmg.a.61191

Pannicke U, Hönig M, Hess I, Friesen C, Holzmann K, Rump EM, Barth TF, Rojewski MT, Schulz A, Boehm T, et al. 2009. Reticular dysgenesis (aleukocytosis) is caused by mutations in the gene encoding mitochondrial adenylate kinase 2. Nat Genet 41: 101-105. doi:10.1038/ng.265

Pesaran T, Karam R, Huether R, Li S, Farber-Katz S, Chamberlin A, Chong H, LaDuca H, Elliott A. 2016. Beyond DNA: an integrated and functional approach for classifying germline variants in breast cancer genes. Int $J$ Breast Cancer 2016: 2469523. doi:10.1155/2016/2469523

Reese MG, Eeckman FH, Kulp D, Haussler D. 1997. Improved splice site detection in Genie. J Comput Biol 4: 311-323. doi:10.1089/cmb.1997.4.311

Richards S, Aziz N, Bale S, Bick D, Das S, Gastier-Foster J, Grody WW, Hegde M, Lyon E, Spector E, et al. 2015. Standards and guidelines for the interpretation of sequence variants: a joint consensus recommendation of the American College of Medical Genetics and Genomics and the Association for Molecular Pathology. Genet Med 17: 405-424. doi:10.1038/gim.2015.30

Schafer S, Miao K, Benson CC, Heinig M, Cook SA, Hubner N. 2015. Alternative splicing signatures in RNA-seq data: percent spliced in (PSI). Curr Protoc Hum Genet 87: 11.16.11-11.16.14. doi:10.1002/0471142905 .hg1116s87

Six E, Lagresle-Peyrou C, Susini S, De Chappedelaine C, Sigrist N, Sadek H, Chouteau M, Cagnard N, Fontenay M, Hermine $\mathrm{O}$, et al. 2015. AK2 deficiency compromises the mitochondrial energy metabolism required for differentiation of human neutrophil and lymphoid lineages. Cell Death Dis 6: e1856. doi:10 1038/cddis.2015.211

Yeo G, Burge CB. 2004. Maximum entropy modeling of short sequence motifs with applications to RNA splicing signals. J Comput Biol 11: 377-394. doi:10.1089/1066527041410418 


\section{COLD SPRING HARBOR Molecular Case Studies}

\section{Reticular dysgenesis caused by an intronic pathogenic variant in AK2}

Shoji Ichikawa, Susan Prockop, Charlotte Cunningham-Rundles, et al.

Cold Spring Harb Mol Case Stud 2020, 6: a005017

Access the most recent version at doi: $10.1101 / \mathrm{mcs} . a 005017$

License This article is distributed under the terms of the Creative Commons

Attribution-NonCommercial License, which permits reuse and redistribution, except for commercial purposes, provided that the original author and source are credited.

Email Alerting Receive free email alerts when new articles cite this article - sign up in the box at the Service top right corner of the article or click here. 